HAUSGEMEINSCHAFTEN

\section{Alternativen sind möglich}

Kaspar Pfister

Hausgemeinschaften gelten in der Altenpflege als Modell der Zukunft. Derzeit stellen sich allerdings noch manche konzeptionelle, rechtliche und wirtschaftliche Fragen. Ein Modell in Baden-Württemberg zeigt, wie es gehen kann.

Mitte des letzten Jahrzehnts entstanden in Deutschland die ersten Hausgemeinschaften in der stationären Heimstruktur. Ziel dieses neuen Angebots ist die Verbesserung der Lebensqualität älterer, pflegebedürftiger Menschen, die im Heim leben. Verschiedene, angeblich unabänderbare Bedingungen verursachen bei den bisherigen, vor allem frei finanzierten Projekten nicht selten wirtschaftliche Probleme. Dies mag einer der entscheidenden Gründe sein, warum sich diese Konzeption noch nicht überall durchsetzen konnte.

Derzeit herrscht eine Euphorie im Bau von Pflegeheimen, wobei neben sinnvollen Projekten zu viele Vorhaben realisiert werden, die am Bedarf vorbei gehen - sei es quantitativ oder qualitativ. Eine Reihe von neuen Einrichtungen nennt sich dabei aus Marketinggründen Hausgemeinschaftskonzept, die meisten sind davon jedoch weit entfernt.

Im Süden Deutschlands wurde in der Stadt Mössingen im Landkreis Tübingen im Oktober vergangenen Jahres von dem privaten Betreiber »BeneVit« ein Pflegeheim - mit der Bank für Sozialwirtschaft frei finanziert nach dem Hausgemeinschaftskonzept eröffnet, das diese Konzeption konsequent und erfolgreich umsetzt.

Der Neubau besteht aus sechs Wohnungen mit jeweils zwölf Betten, aufgeteilt auf drei Etagen. Jede
Wohngruppe verfügt über eine eigene Küche, Essraum, Wohnzimmer, Nebenräume wie Abstellkammern, Arbeitsräume usw. Die Zimmerstruktur besteht aus 46 Einzelzimmer und 13 Doppelzimmer. Sämtliche Nebenräume wie Mehrzweckraum, Therapieraum, Lagerräume, Personalaufenthaltsräume, Pflegebäder usw. sind vorhanden. Alle Tätigkeiten finden im Haus statt. Es gibt keine ausgelagerten Dienste - alles wird in der Wohnung; erledigt. Der Tagesablauf wird durch Präsenzkräfte gestaltet auch das gemeinsame Zubereiten der Mahlzeiten, die Reinigung, die Wäscherei usw.

Die komplette Essenszubereitung erfolgt täglich dezentral in jeder Wohnung, in jeder Wohngruppe kann also etwas anderes gekocht werden. Dabei wird der Speiseplan mit den Bewohnern jeder Wohnung individuell besprochen und nicht selten am Morgen noch mal umdisponiert. Die Bewohner sind mit dabei, wenn es um die Zubereitung der Mahlzeiten geht und sie sind in die Alltagsarbeiten eingebunden. Im Wohnzimmer jeder Wohnung steht neben den Sesseln das Sofa und in der kalten Jahreszeit spendet der Kaminofen wohlige Wärme und das Prasseln des Feuers ist nicht nur zum Anschauen schön.

\section{Wie die Pflege funktioniert}

Die Planung und Durchführung der Pflege erfolgt - ähnlich wie im ambulanten Bereich - durch einen zentralen hauseigenen Pflegedienst für die gesamte Einrichtung. Dabei ist der Arbeitsplatz der Pflege vom Medikamentenschrank bis hin zum Schreibtisch in das Wohnzimmer jeder Wohnung integriert.

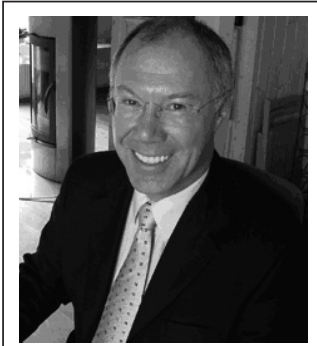

Kaspar Pfister ist gelernter DiplomVerwaltungswirt und war lange Jahre als Verwaltungsbeamter im Kommunalbereich tätig, zuletzt als Kämmerer und stellvertretender Bürgermeister. Von 1996 bis 2002 war er als Kaufmännischer Geschäftsführer der St. Anna Hilfe für ältere Menschen der Stiftung Liebenau und der Salvator Altenhilfe gGmbH in Baden Württemberg verantwortlich für 27 stationäre Einrichtungen mit Dauer- und Kurzzeitpflege, für 18 Wohnanlagen, ambulante Dienste, geriatrische Rehabilitationsklinik, Pflegehotel mit insgesamt 1.640 Mitarbeiterinnen und Mitarbeitern. Seither ist er geschäftsführender Gesellschafter der BeneVit, Pflegemanagement \& Consulting $\mathrm{GmbH}$ und Geschäftsführer der BeneVit, Vorarlberger Pflegemanagement gGmbH, jeweils Sitz in Dornbirn, Österreich mit drei Tochtergesellschaften in Deutschland und einer Gesellschaft in Spanien.

Internet http://www.benevitpflege.de

Der gesamte Personalbedarf bemisst sich nach den Personalschlüsselvereinbarungen mit den Pflegekassen; der Einsatz wird jedoch anders organisiert. Da alles Personal, das über den Pflegesatz finanziert wird, im Haus direkt in der Wohnung tätig ist, erhöht sich die Quote der beim Bewohner tätigen Mitarbeiterschaft um rund 40 Prozent - und dies ist deutlich in der Qualität spürbar.

Aufnahme im Haus finden alle Menschen, die eine stationäre Struktur benötigen, von der Pflegestufe 0 bis 3. Das Konzept ist nicht nur, aber auch, für demenziell erkrankte Menschen geeignet. 
Das oftmals anscheinend größere Problem der Wirtschaftlichkeit der Hausgemeinschaftskonzepte stellt sich in dieser Einrichtung nicht; die vereinbarten Entgelte betragen:

- Stufe 0: 72,55 Euro je Tag

- Stufe 1: 83,45 Euro je Tag

- Stufe 2: 97,95 Euro je Tag

- Stufe 3: 115,65 Euro je Tag

- Für Selbstzahler entsteht ein Aufschlag von zwei bis drei Euro am Tag, je nach Zimmertyp.

Da alle Leistungen von den Mitarbeiterinnen und Mitarbeitern des Hauses selbst erbracht werden, entstehen wirtschaftliche Vorteile, die den höheren Personalaufwand ausgleichen.

Die angeblichen Vorteile des Outsourcings - vor allem von hauswirtschaftlichen Tätigkeiten - wurden im Vorfeld besonders analysiert. Ergebnis: Bei der Beauftragung eines externen Dienstleisters entstehen bei gleichen Qualitätsansprüchen nur dann Vorteile, wenn die eigenen Personalkosten überdurchschnittlich hoch sind. Dabei wird oft unterschätzt, dass neben der Umsatzsteuer auch der Gewinn des Dienstleisters kompensiert werden muss. Jedenfalls zeigt sich bei diesem Praxisbeispiel, dass die eigene Leistungserbringung nicht zwangsläufig teuerer sein muss. Da alle anfallenden Tätigkeiten mit den Bewohnerinnen und Bewohnern erfolgen, wird gleichzeitig ein therapeutischer Effekt durch die Natürlichkeit des Alltagsgeschehens erzeugt. Obwohl dieses Mitwirken der Bewohnerschaft für die Mitarbeiterinnen und Mitarbeiter zeitintensiver und aufwendiger ist, zeigen sich anderweitige, deutliche Vorteile:

- Das Hausgemeinschaftsmodell ermöglicht durch eine an der Normalität orientierte Tagesstruktur die Erhaltung, Stärkung oder Reaktivierung der Alltagskompetenz der Bewohnerinnen und Bewohner.

- Der Tagesablauf der Bewohnerinnen und Bewohner wird nicht durch medizinische, pflegerische Notwendigkeiten dominiert.

- Durch die Einbindung der Bewohnerschaft in Alltägliches wird deren Selbstverantwortung gefördert und gestärkt.

- Die tendenzielle Begrenzung der Zunahme der Pflegebedürftigkeit der Bewohner bis hin zu deutlichen Verbesserungen führen zu einer finanziellen Entlastung der Selbstzahler und der öffentlichen Hand (Sozialhilfe und Pflegekassen). In der Praxis zeigt sich oft der Effekt, dass vermeintliche höhere Pflegebedürfnisse sich nach wenigen Wochen deutlich reduzieren und angenommene Einstufungserwartungen zurückgeschraubt werden können.

Die Baukosten sind vergleichbar mit konventionellen Einrichtungen, allerdings ist die Raumstruktur völlig anders verteilt. Von außen sieht das Haus aus wie andere Mehrfamilien- häuser oder normaler Geschosswohnungsbau. So mancher Besucher, der zum ersten Mal kommt, fährt oder geht am Haus vorbei, weil er eine Pflegeheim sucht und das große mehrgeschossige Wohnhaus als solches nicht wahrnimmt.

Die Genehmigungsphase gestaltet sich oftmals schwierig. Bei diesem wie bei anderen Projekten hat sich jedoch herausgestellt, dass die von »BeneVit « erstellten Expertisen unter Beiziehung von verschiedenen, externen Experten hinsichtlich Brandschutz, Hygiene usw. letztendlich Behörden überzeugen.

\section{\begin{tabular}{|c|}
\hline \\
\hline Hausgemeinschaftsmodell \\
Eigenschaften
\end{tabular}}

1. Normalitätsprinzip

2. Überschaubare Wohngruppen mit 12 bis 14 Bewohnern

3. Tagesablauf wird mit der Präsenzkraft gestaltet

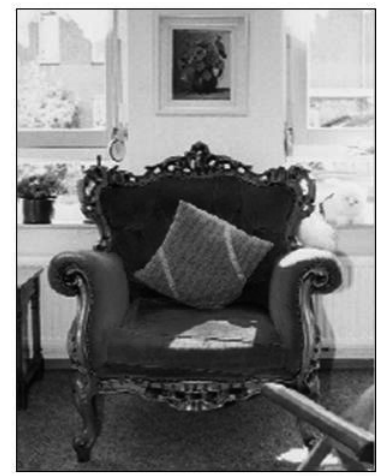

4. Kochen in Wohngruppen

5. Pflege wird unter ambulantem Aspekt organisiert

6. Zurücknahme der Pflegedominanz
Hausgemeinschaftsmodell Vorteile
1. Keine Hospitalisierung

2. Verbesserung der Lebensqualität

3. Förderung der Ressourcen
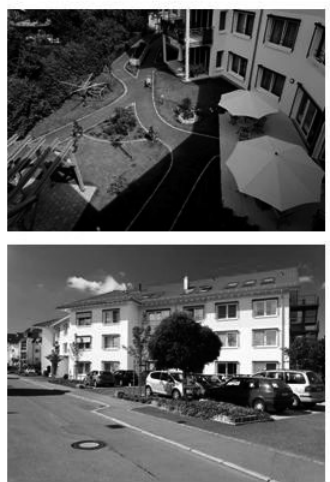

4. Verbesserung des Allgemeinzustands

5. Förderung der Selbständigkeit und Eigenverantwortung für den letzten Lebensabschnitt

6. Kostenneutral 
Täglich ist zu beobachten, wie Menschen mit dieser Konzeption neue Lebensfreude, Erfolg und Spaß erfahren und neu motiviert werden. Enttäuschung, Konflikte und Sterben sind dabei auch Alltag und werden gemeinsam bewältigt. Die Bandbreite des Erlebten spiegelt in hohem Maß den normalen häuslichen Alltag wieder und ist deutlich breiter gefächert, wie dies in konventionellen Konzepten möglich ist.

Die Anforderungen an Mitarbeiterinnen und Mitarbeiter sind anders zu definieren. Für Präsenzkräfte und Pflege bedeutet ein Arbeiten in dieser Konzeption eine neue Ausrichtung, kommt aber den Erwartungen der Mitarbeiterinnen und Mitarbeiter an ein sinnvolles Arbeiten näher. Jeden-
Kinder sind da, seien es Nachbarkinder, die nach den Hasen im Garten schauen oder die Kinder der Mitarbeiter, die nach der Schule mit der Mama mitgehen und nur da sind oder Spiele spielen oder im Rosencafé sitzen und im Internet surfen.

Mitarbeiter gehen mit Bewohnern mal ins Eiscafé oder auf den Wochenmarkt und kaufen ein, was man halt so für den Haushalt braucht. Die Beispiele lassen sich unendlich fortsetzen und die Pflegeleitung stellt nach 30-jähriger Berufserfahrung fest, dass trotz aller Qualität in den vorigen Einrichtungen, in denen sie tätig war, nur ein Bruchteil dessen geschehen ist, was täglich im »Haus Blumenküche« erlebt wird.

\section{»Outsourcing rechnet sich nur, wenn die eigenen Personalkosten überdurchschnittlich hoch sind "}

falls liegen auch im arbeitspolitisch unproblematischen Süden Deutschlands ohne Ausschreibung genügend Bewerbungen für alle Bereiche vor, auch von guten Fachkräften.

Erfreulich ist die Reaktion der Angehörigen. In konventionelle Einrichtungen gehen sie oft mit schlechtem Gewissen und selten. In die Wohngruppe kommen sie gern, sind Gast und fühlen sich wohl. Das schlechte Gewissen, den Angehörigen aus welchen Gründen auch immer nicht zu Hause betreuen zu können, ist deutlich reduziert. Man spürt, dass in diesem Haus in hohem Maß Spaß und Lebensfreude vorhanden ist.

Besucher kommen zum Besichtigen fast täglich nicht nur aus Deutschland ins Haus und jeder geht mit der Überzeugung, dass für einen selbst eine solche Wohnform im Alter gut vorstellbar wäre. Erstaunt zeigen sich Besucher, dass es nicht nach »alten Menschen riecht «. Dafür riecht es nach Kaffee, Mittagessen, frisch gebackenen Kuchen (der jeden Tag in jeder Wohnung frisch aus dem Ofen kommt) und man hört lachen und singen.

\section{Resümee}

Ist Demenz heilbar? Die Mediziner mögen sich darüber streiten; aus heutiger Sicht vielleicht nicht, aber die demenziell erkrankten Bewohnerinnen und Bewohner sind nicht mehr wieder zu erkennen, sie haben eine Tagesstruktur, gehen spazieren, sind beschäftigt und leben den Alltag.

Ohne dieses Konzept ist aber auch schnell spürbar, wie schnell die Desorientierung wieder in den Vordergrund tritt, wenn man nach der Kurzzeitpflege wieder in der häuslichen Umgebung kommt und doch wieder viele Stunden am Tag zu Hause allein ist.

Entscheidend für den Erfolg solcher Konzepte ist die Summe der Details, die alle stimmen und zusammen passen müssen. Wenn dies gelingt, können Wohngruppen gute Beispiele für stationäres Wohnen bei Pflegebedürftigkeit sein. Jedenfalls ein Haus in dem Bewohner, Angehörige und Mitarbeiter sich wohl fühlen und in das Gäste und Besucher gerne kommen.
Soziale Dienste in Europa

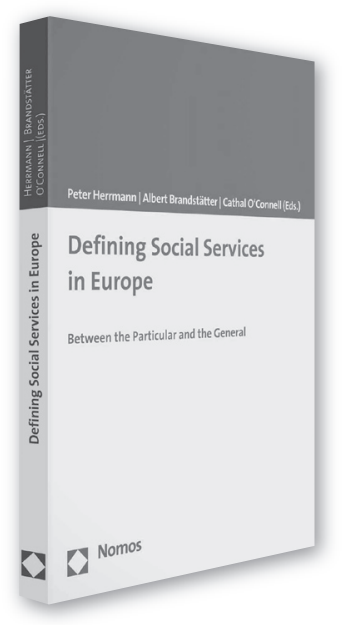

Defining Social Services in Europe

Between the Particular and the General

Herausgegeben von Dr. Peter Herrmann, European Social, Organisational and Science Consultancy, Albert Brandstätter und Dr. Cathal O‘Connell, University College Cork, Ireland

2007, 276 S., brosch., 44,- $€$, ISBN 978-3-8329-2883-4

Soziale Dienstleistungen des Allgemeinen Interesses stehen hoch im Kurs der EU-politischen Debatte um die Wettbewerbsfreiheit. Aber können Dienste für jene, die aus dem Wettbewerb fallen, überhaupt dem Wettbewerbsrecht folgen? Und leisten sie nicht einen Dienst gerade auch für die Gesellschaft, anstatt nur für hilfebedürftige Individuen da zu sein?

Der Band untersucht verschiedene Aspekte der Qualität sozialer Dienste aus theoretischer und praktischer Sicht.

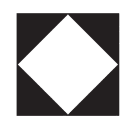

Nomos

Bitte bestellen Sie bei Ihrer Buchhandlung oder bei Nomos | Telefon 07221/2104-37 | Fax -43 | www.nomos.de | sabine.horn@nomos.de 